\title{
THE CONCEPT OF PURPOSEFUL PROTOTYPING: TOWARDS A NEW KIND OF TAXONOMIC CLASSIFICATION
}

\author{
Petrakis, Konstantinos; Hird, Abigail; Wodehouse, Andrew \\ University of Strathclyde
}

\begin{abstract}
A prototype can be generally defined as a preliminary version of a final product and it can represent both aesthetic and functional features. Prototyping, the process of building a prototype, constitutes an indispensable part of product development processes. Many classifications of prototypes which use a different range of criteria have already been developed and are known as Prototype Taxonomies. This paper proposes an agenda towards the development of a new taxonomic classification which will be based on a prototype's attributes and its intended purpose. This work is justified from the results of an analysis of 8 existing prototype taxonomies which indicate that there is not a taxonomy that explicitly uses the prototypes' purposes as its basic taxonomic dimension. A definition of the term 'Prototype Purpose' is proposed through discussing the differences and relations to the term 'Prototype Role'. This work results in a list of 23 explicit prototype purposes which arise from 7 roles of prototyping found in relevant literature: Learning, Communication, Demonstration, Integration, Refinement, Exploration and Requirement Elicitation.
\end{abstract}

Keywords: Design engineering, Design process, Prototypes, Purposes, Design methods

\section{Contact:}

Petrakis, Konstantinos

University of Strathclyde

Design, Manufacture \& Engineering Management

United Kingdom

konstantinos.petrakis@strath.ac.uk 


\section{INTRODUCTION}

According to English Oxford Dictionaries, a prototype is "A first or preliminary version of a device or vehicle from which other forms are developed". The etymology of the word originates in the Greek language where the word "prototypon" which can be analysed in two terms: "protos" (first) + "typos" (form/impression). In engineering design, many definitions of what constitutes a prototype can be found in the literature. It can be defined as "an approximation of the product along one or more dimensions of interest" (Ulrich and Eppinger, 2012), or "an artefact that approximates a feature (or multiple features of a product, service, or system" (Otto and Wood, 2001). As seen in Figure 1, prototypes can take several forms and can be made of a wide range of materials. Other definitions state that any type of model or design representation that embodies aspects of functionality and look and feel of the final product can be considered as a prototype, including sketches and rough models (Gurjar, 2015). According to others, the main differences between a prototype and a design concept are the fact that the prototype can be tested, in comparison with a simple idea (Camburn et al., 2017). Relevant research has also defined prototypes by using their role as a basis, namely as representations of information (Ullman, 2002) and "physical or digital embodiment of critical elements of the intended design, and an iterative tool to enhance communication, enable learning, and inform decision-making at any point in the design process" (Lauff et al., 2018). For the purpose of this research, which is focusing on the purposes of prototypes, we will be mainly based on the above definition that is derived upon the emergent roles of prototypes.

The overall objective of this research is to develop a new taxonomic classification of prototypes which will be based on the mapping between prototypes' attributes and intended purpose, in order to assist designers in building the right prototype for the right purpose. This paper presents an agenda towards the development of this taxonomy by presenting a list of purposes, arising from prototype roles, which can act as an initial set of guidelines. In Chapter 2, existing prototype taxonomies are discussed and analysed according to the grouping criteria used. Chapter 3 focuses on the development of the 'Purposeful Prototyping' concept, and through the review of prototyping's fundamental roles, a list of growing prototyping purposes is developed. The last chapter concludes this work and provides some insights regarding the next steps of this research.

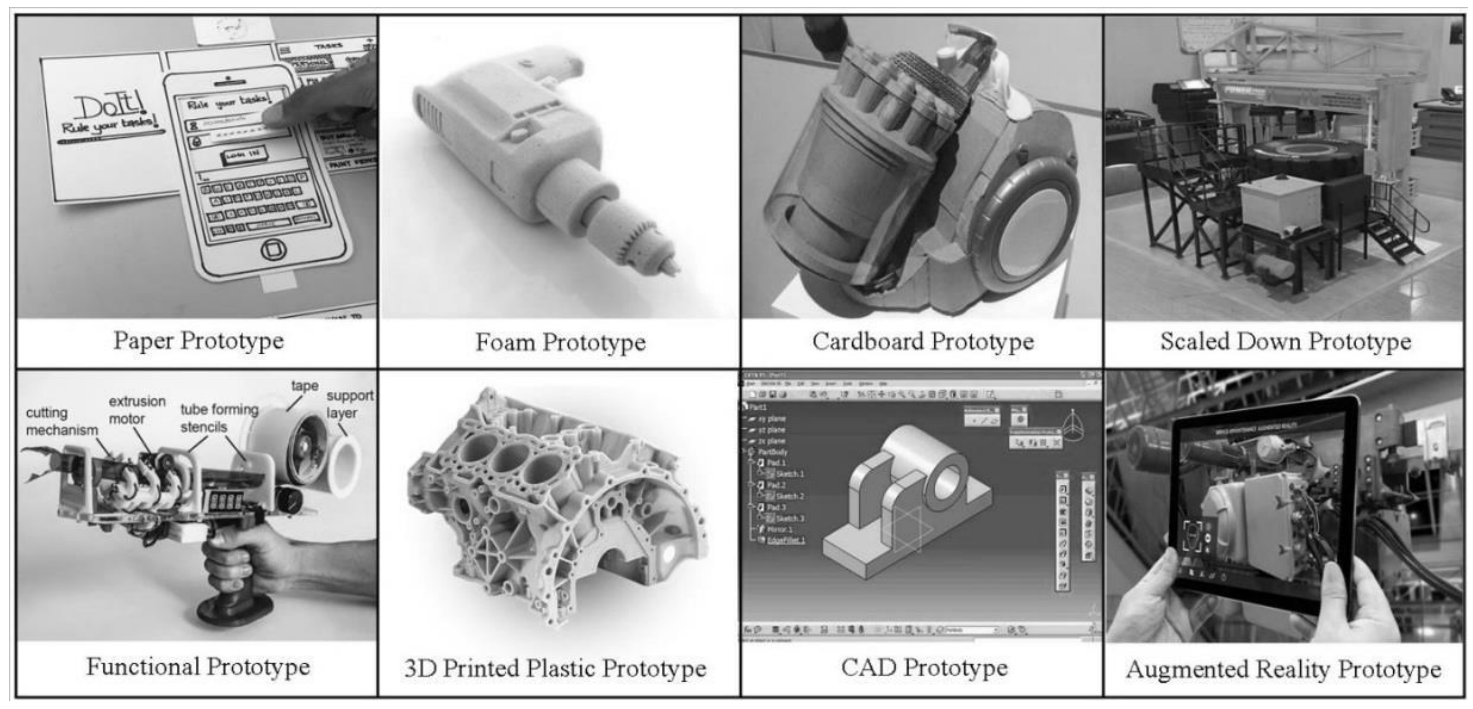

Figure 1. Range of different types of Prototypes

\section{PROTOTYPE TAXONOMIES: LITERATURE REVIEW AND ANALYSIS}

There are many efforts in trying to classifying prototypes into groups according to a range of measures. These are widely known as Prototype Taxonomies and are considered beneficial for designers by acting as decision-support and planning tools. Taxonomy is the science that is concerned with the classification and organisation of data (Michaelraj et al., 2008). In general, the development of such Prototype Taxonomies enhances the understanding about prototypes, assists in the development of a common communication language between designers and therefore facilitates them 
to prototype more effectively (Pei et al., 2015). For the purpose of this paper, the eight most relevant and discussed taxonomic classifications of prototypes were selected and are analysed and discussed in Section 2.1. The most prominent of grouping criteria found are related to whether prototypes represent form or functionality, their level of fidelity in comparison to the final product, type of media used for their representation (namely physical or virtual), their level of requirement relaxation, evaluation purpose as well as the phase of the design in which their usage is applied. A table is also created in order to show the taxonomic criteria by which each classification is developed. The taxonomies are presented in chronological order, from the oldest to the most recent. The mapping is performed according to the level by which examined taxonomies use each classification criteria; the symbol $(\bullet)$ means that the taxonomy explicitly uses these classification criteria, whereas the symbol (o) shows that the classification criteria are used to a lower degree. Blank cells indicate that the taxonomy does not consider these classification criteria.

\subsection{Existing taxonomic classifications of prototypes}

1. To begin with, Otto and Wood (2001) focus only on physical prototypes and use six different classes: Proof of Concept Models, Industrial Design Prototypes, Design of Experiments Prototypes, Alpha Prototypes, Beta Prototypes and Preproduction Prototypes. Although there is some mentioning to the way that each prototype should be used or what kind of questions it may answer, this classification is mainly based on the evaluation purpose of its prototype as well as the level of fidelity as opposed to the final product, however in terms of materials rather than the level of requirement integration.

2. Another classification that is built according to evaluation purpose is done by Ullman, (2002) and classifies prototypes into Proof-of-Concept, Proof-of-Product, Proof-of-Process and Proof-ofProduction depending on the measures that they verify when used, namely function, geometry, tooling etc. This classification is also based on the stage of the design process as well as on the level of Requirement relaxation to a lower degree.

3. The taxonomy proposed by Lim, Stolterman and Tenenberg (2008), is a totally different kind of classification, referred to as Anatomy of Prototypes and aims to assist designers in exploring the design space rather than only evaluating their ideas. Prototypes are divided into Filters and Manifestations of Ideas mainly depending on their level of fidelity and the way that they are presented, without specifying either physical or virtual prototypes.

4. In another proposed taxonomy which focuses on physical prototypes, just as the majority of the discussed classifications, the two main groups are Factors and Characteristics of a prototype (Michaelraj et al., 2008). Factors include Evaluation Purpose, Communication and Design Stage and Characteristics include Size, Type, Material and Fabrication. Communication, a major purpose of prototyping, is involved as a taxonomic factor and the researchers specifically note the various purposes of prototyping in the product development process which in turn raise the need for this taxonomy. However, in our opinion their taxonomy is more focused on the characteristics of prototypes in terms of form and function rather than their intended purposes.

5. The Hierarchical Morphological Prototype (HMP) Taxonomy (Stowe, 2008) is exclusively depending on the characteristics of a prototype and defines prototypes according to three dimensions: Variety (which classifies them into Physical and Non-physical), Complexity (the level of system integration namely component, system or sub-system) and Fidelity (the level of resemblance to the final product). The main benefit of this taxonomy is that it can incorporate any feasible prototype quickly and without much effort, without focusing only on physical models, however it does not consider any prototyping purposes as a basis, making it a less useful tool during the establishment of a potential prototyping strategy.

6. The valuable study conducted by Pei, Campbell and Evans, (2015) covers multiple kinds of visual design representations (VDRs) and includes prototypes as the 4th group along with Sketches, Drawings and Models. For the purpose of this work, focus is only given to Models and Prototypes, which are distinct according to their evaluation purpose and stage in the process. Industrial Design Prototypes are described as detailed three-dimensional full-scale representations that include both functional and aesthetic features and are made from the actual material or a simulation of it. Engineering Design Prototypes are divided into seven subcategories based on their level of fidelity and level of system integration, namely Experimental, Alpha, Beta, System, Final Hardware, Off-Tool Components and Pre-Production prototypes. 
Although this taxonomy is truly descriptive and easy to use, it concentrates more on the representations' features. Although, accompanied with visual images as examples, some attention is also given to the prototypes' intended purpose in their description, making it a respected tool for supporting design decisions.

7. Ulrich and Eppinger (2012) taxonomy is built depending on the level of fidelity, requirement relaxation and the way that each prototype is presented. They classify prototypes along two basic dimensions. The first dimension examines how much the prototype is physical or analytical, namely tangible artefacts with certain feature of the product or nontangible product representations such as computer simulations or mathematical models, respectively. The second dimension has to do with how much the prototype is comprehensive rather than focused. Comprehensive prototypes carry the majority of the product's most significant attributes, whereas focused prototypes are used with the aim of examining one or a few specific product characteristics.

8. Lastly, Isa and Liem (2014) focus solely on physical models and develop their taxonomy mostly based on level on fidelity and requirement integration and less on whether the prototypes address form or function. Soft and Hard models are described as non-functional models whereas Presentation models and Prototypes are functional representations that include the most important specifications of the product.

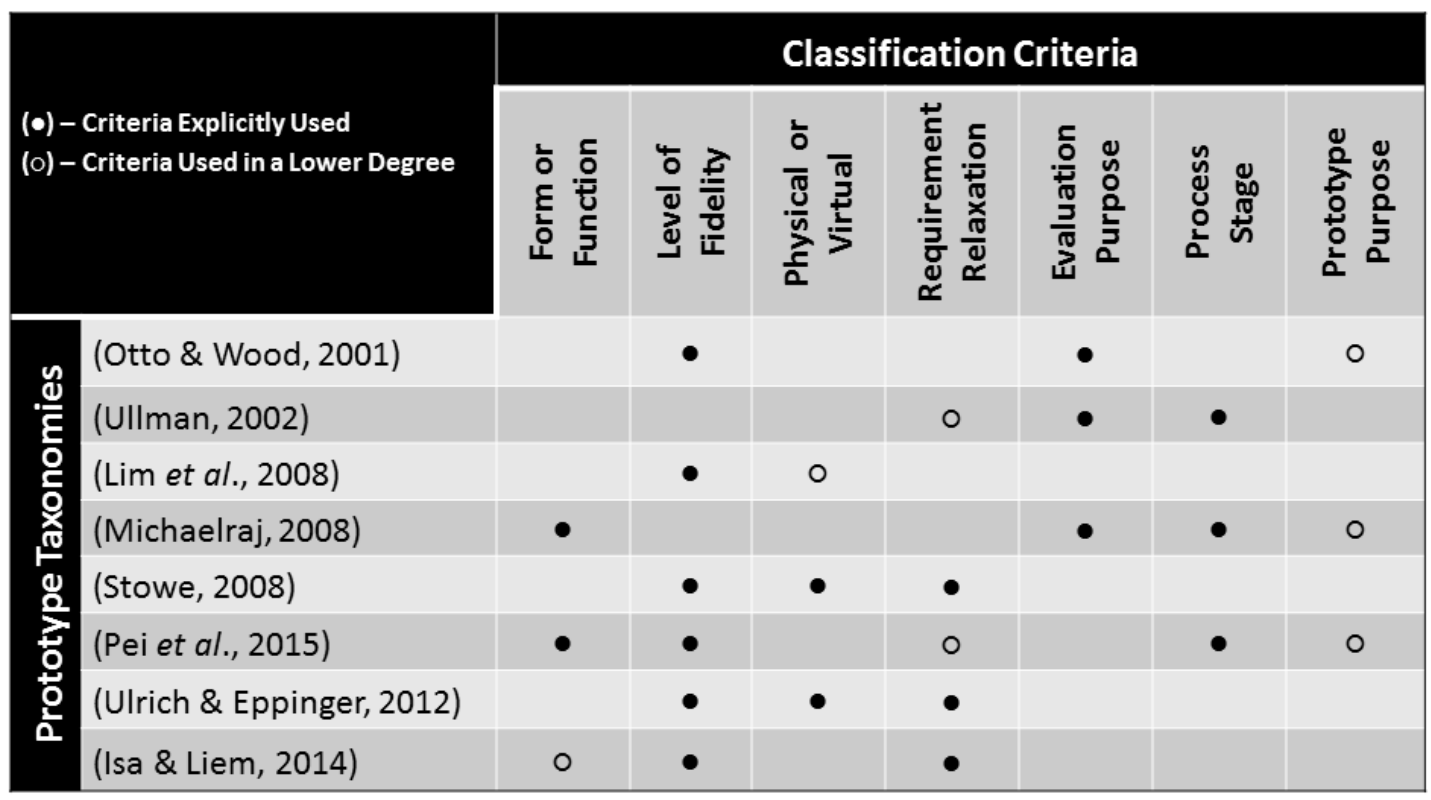

Figure 2. Mapping of prototype taxonomies and their classification criteria

\subsection{Findings based on the mapping between taxonomies and criteria}

There are four findings that arise from the conducted analysis of the prototype taxonomies found in the literature as well as the mapping between the criteria used in each taxonomic effort:

- Firstly, half of the discussed taxonomies put their focus exclusively into physical models or prototypes (Otto and Wood, 2001; Ullman, 2002; Michaelraj et al., 2008; Isa and Liem, 2014). In the case of the VDR Taxonomy several types of representations are covered. However, as far as prototypes are concerned they are treated as three-dimensional representations that are often build to full size (Pei et al., 2015). Only two of the included taxonomies make a clear distinction between physical and non-physical prototypes (Stowe, 2008; Ulrich and Eppinger, 2012), whereas Lim et al., (2008) state that the performance of an idea through a prototype can take any form, shape or appearance.

- Secondly, 'Level of Fidelity', which represents how much prototypes bear a resemblance to the final product, appears to be the most common taxonomic criterion amongst the examined classifications. This is quite natural if we consider that it is one of the main factors that describe a prototype and many of the definitions given to the term 'Prototype' talk about approximation to the final product (Otto and Wood, 2001; Ulrich and Eppinger, 2012). 
- As it is also seen in Figure 2, there are strong relationships between the criteria 'Level of Fidelity' and 'Requirement Relaxation', as most of the taxonomies that fall into one group also fall into the other. This is logical because the prototype's level of resemblance to the final model is definitely depending on how many of the defined requirements are incorporated into it. However, these two criteria should not be confused as in this case 'Level of Fidelity' is mainly related to the prototypes aesthetic and look-and-feel features, whereas the level of Requirement Relaxation has to do more with functionality aspects. For example, in Ullman's proposed taxonomy there is reference about the prototype's requirements according to usage and the design stage, nevertheless it does not talk about high or low fidelity with respect to the final model (Ullman, 2002).

- Lastly, and most importantly, it is found that even a few of the proposed taxonomies that were discussed are taking the prototypes' purpose or role into account (Ullman, 2002; Michaelraj et al., 2008; Pei et al., 2015), there is a clear lack of a classification that explicitly uses the purpose of creating a prototype as its basic taxonomic dimension. Therefore, the presented analysis highlights the need of a different kind of taxonomic classification that will explore the relationships between a prototype's characteristics and its intended use.

\section{THE “PURPOSEFUL PROTOTYPING” CONCEPT}

In order to properly develop this concept we must first discuss the meaning of a prototype's 'role' and therefore examine the relationships between these two terms. A prototype's role can be defined in general as its expected behaviour and function (Otto and Wood, 2001). In the product development process, prototyping's broader role is to minimise risk and uncertainty and its ultimate goal is to enhance performance and overall user experience of the final product (Camburn et al., 2017). Moreover, the actual process of building the prototype can also provide valued understandings to the designers (Ullman, 2002; Hess and Summers, 2013). According to Stowe (2008), it is hard to explicitly define the role of a prototype since its role is not its only defining characteristic and also because a single prototype can have multiple roles at a time. These can be decided by a number of factors such as the project's needs, the manufacturing method of the prototype or the specific phase of the process. The most prominent roles found in the literature are Learning, Communication, Demonstration and Integration. Other types also mentioned but less explored are Design Refinement, Exploration and Elicitation of Requirements. All of these different roles of prototypes will be discussed in sub-section 3.1 below.

As far as the term 'purpose' is concerned, this study will be based on the actual terminology of the word; in Oxford Dictionaries the word 'purpose' is defined as 'the reason for which something is done or created or for which something exists'. Therefore we refer to the term 'prototype purpose' as the actual reason of building a prototype in order to achieve explicit, already set objectives. Sometimes a prototype's purpose is also regarded as one of its key characteristics which should be carefully considered when using it (Ullman, 2002). However this study focuses on the mapping between the prototypes physical features and its intended purpose with the aim of constructing a new taxonomy. The concept of 'purposeful prototyping' can be regarded as the initiation of a prototyping activity through which the prototyping outcome will enable the achievement of specific design objectives. 'Design is the evolution of information punctuated by decisions' (Ullman, 2002), and prototypes can be also defined as the embodiment of such information. Therefore the prototyping outcome as well as the process of creating the prototype itself can provide significant insights and diminish uncertainty related to these decisions. In more detail, interaction with prototypes can inform design decisionmaking regarding the desirability, viability and feasibility of the product. Such decisions may involve both informal, daily decisions related to refinement of the product and formal decisions made for confirming the forward progress of the whole project (Lauff et al., 2018) and are encompassed within all fundamental roles of prototyping. The difference between the terms 'role' and 'purpose' is mainly based on the definitions of 'expected function' and 'intended use', respectively. Consequently, for the sake of the Purposeful Prototyping concept specific focus is given to the establishment of the prototype's purpose, prior to initiating the prototyping process. The activity of defining this purpose requires reflective thinking which comes less naturally to novice engineering designers or students, therefore there is a clear need for a taxonomic classification that addresses this issue. 


\subsection{Literature review of prototype roles}

Learning: During the product development process prototypes can commonly assist in learning by providing various types of new information to all stakeholders by reinforcing their already gained knowledge, as well as by discovering unknown and unexplored information (Lauff et al., 2018). More specifically, prototypes can operate as learning tools in terms of answering specific questions regarding the functionality of the product or by measuring the level by which the product satisfies the customers' needs (Ulrich and Eppinger, 2012). Using prototypes enables to focus on ideas and therefore judge their feasibility by analysing a total of measurements which may also lead to unveiling unpredicted phenomena and detecting potential problems (Otto and Wood, 2001; Hess and Summers, 2013). This kind of unknowns can also include concept selection issues, apart from functionality and feasibility (Stowe, 2008). Prototypes are also referred to as learning catalysts during the development of new products, offering the chance to better understand the anticipated critical functions of a product (Menold et al., 2017). According to a recent study, it is found that engineering designer teams of all levels, in both academic and industrial sectors, manage to accomplish higher technical functionality by using prototypes early in the process, as they were able to identify shortcomings a lot quicker and therefore adjust their concepts accordingly in order to achieve higher quality products (Elverum and Welo, 2014). However, others argue that engineering uncertainties are reduced during the latter stages of the process, whereas prototypes used in earlier stages assist in the visualisation of ideas and in the clarification and disambiguation between potential concepts (Lande and Leifer, 2009). Learning through prototyping activities can also happen by interacting or referencing prototypes (Lauff et al., 2018). Apart from information related to technical elements and users' behaviours and preferences, prototyping assists in learning about the design space associated with a product, namely by getting to familiarise with existing technologies and benchmarking competitors' products. By providing answers to questions regarding feasibility, desirability and viability they can also enhance designers' tacit knowledge of a product type. This knowledge may also encompass more business-related matters, namely materials' costing, manufacturing and tooling which derive upon iterative testing and interaction with prototypes (Lauff et al., 2018). Even in the case of a prototype failing to work as planned or to achieve its intended purpose, it is still able to provide significant information and indicate the mistakes that should be avoided in the future during its fabrication. Consequently, prototyping failure can also be considered as a learning opportunity which aims to improve designers' technical knowledge as well as fabrication abilities (Gerber and Carroll, 2012).

Communication: When prototypes are used during the process they act as methods of communication mainly between the designers and the rest of the project's stakeholders such as managements, investors, superiors, clients and of course end users, as they can present and share information regarding the product's features. The transported information may be about design functionality, performance, project understanding or the product's look and feel (Stowe, 2008). Especially physical prototypes, but also three-dimensional visual representations are able to transfer a more understandable kind of knowledge regarding the product's aesthetic and spatial features in contrast with verbal descriptions or sketches of the same product (Ulrich and Eppinger, 2012). The communicated information is especially important for designers because they gain feedback from all those involved in the product development process with the purpose of refining the prototype (Otto and Wood, 2001). The earlier in the process prototypes are used for communication, the sooner a common language between stakeholders is developed, leading to better team dynamics. Prototypes can also facilitate the start of a discussion during a meeting and therefore act as reference points (Hess and Summers, 2013). This combination of verbal description and physical interaction enables clearer communication and decreases the chance of misunderstandings within the team (Lauff et al., 2018). Through the sharing of prototype information designers are also facilitated in observing the actual interactions between the users and the prototype as well as the interaction among the users when they are engaging with the prototype (Camburn et al., 2017). This is particularly important as far as the involvement of human factors in the design process is concerned. Users' emotions are strongly affected by the fidelity of prototypes; high fidelity leads to positive behaviours. Thus, prototypes that facilitate the engagement of designers and users lead to more valuable insights and understanding (Menold et al., 2017). Apart from concept explanation, feedback and negotiations, (Lauff et al., 2018) also include 'persuasion' within the four major communication purposes of using prototypes. In more detail they are referred to as "priming tools" which have the ability to revise decision making made by multiple stakeholders if they are presented properly. Besides external communication purposes, 
prototypes play a very important role in the internal communication of the team, namely amongst the designers. They can be used for evaluating everyone's ideas, for convincing the team about the functionality of an idea as well as function as a medium for integration of multiple ideas into a final concept. The appropriate representation of a concept to senior designers or design managers is also vital in order for them to gain a better understanding and not reject a promising design solution (Lande and Leifer, 2009; Elverum and Welo, 2014). This is also verified in a recent study, stating that the sharing of a large number of prototypes within the design team, improves the understanding of the design itself, increases sympathy within the members, increases ideation levels and therefore leads to higher quality design outcomes (Menold et al., 2017).

Refinement: Refinement of a design can be regarded as the process of developing it progressively, until it has reached the intended functions and requirements (Camburn et al., 2017). Prototyping is proved valuable for design refinement as it is capable of identifying design features that have the most impact to the product's performance, it can reveal and decrease errors during fabrication and it can be used for validation of specifications and requirements. According to other definitions, refinement can also be referred to as optimisation of performance, which occurs through a series of testing and experimentation of the part's sub-systems. The results of such experiments lead to informing decisions about various design features with the aim of enhancing the overall performance or aesthetics (Otto and Wood, 2001). Design Refinement can also be considered as a result of prototyping's learning purposes, however it still falls into a separate category as the goals that are set are more specific and mostly related to functionality aspects.

Integration: Prototypes can be used in order to confirm that all the separate parts of a product can fit and work together as intended (Ulrich and Eppinger, 2012). A design team might have created several prototypes of each sub-system and ensured that it has reached its anticipated function, however it is imperative that all of the already tested sub-systems can work together as an assembly. This function is also referred to as Architectural Interfacing, defining it as the testing procedures that ensure all interfaces are compatible within the overall system and perform together properly (Otto and Wood, 2001).

Demonstration: In terms of the planning of the design process, prototypes can be used to set design goals which in turn establish that the product has reached a certain degree of its desired functionality or enforce deadlines that should be touched by a certain time during the process (Ulrich and Eppinger, 2012). These points are commonly known as milestones and are significant markers during the process as they indicate the product's functional progress and therefore allow the whole development project to proceed to the next stage. Otto and Wood, (2001) also highlight the role of prototypes as milestones, referring to these uses as Demonstration and Scheduling. The successful achievement of the already set goals can be demonstrated to both external and internal stakeholders through a prototype. Moreover, prototypes can serve as tools which force taking initial decisions, even during the early design stages, resulting in the benefit of avoiding terminal cycles during the process; such early decisions ensure an up to $60 \%$ decrease of turnaround time, especially when prototypes work as milestone representations comparing to drawings (Otto and Wood, 2001). It is also important to note that when prototypes function as milestones the design teams are enabled to develop an appropriate plan for their process according to their time and cost constraints (Menold et al., 2017). Therefore these milestones set the objectives of the whole team in a way, optimise their product planning and assist them in a more effective type of decision making. Apart from the advantages of prototypes acting as milestones in the successful delivery of the product they also play a positive role in the psychological experience of the design team by creating a sense of forward progress (Gerber and Carroll, 2012). This is also found in a research about engineering design students, where the iteration and continuous refinement of the prototypes that served as milestones proved to be beneficial in motivating them and providing them with valuable guidance (Lande and Leifer, 2009).

Exploration: Design Exploration can be regarded as the process of searching new design concepts and novel kinds of solutions to design problems. There are examples of practicing design teams who are applying the use of prototypes, even during the very early conceptual stages of the design process, in order to explore and test various concepts and therefore minimise their technical uncertainty and reveal unknown problems (Hess and Summers, 2013; Elverum and Welo, 2014). Prototyping's role in concept generation and exploration of the design space has also been linked to the design processes of divergence and convergence, the expanding of multiple generated concepts and the selecting of a smaller set of concepts respectively. However, in terms of design exploration, prototyping's role in idea stimulation 
is more connected to the divergent design activities, as it assists in the discovery of a new set of unknown solutions (Hess and Summers, 2013).

Elicitation: The process of defining the appropriate requirements for a specific product or process is called requirement elicitation. More specifically, apart from collecting requirements it also includes the prioritising of them as well as the identification of the stakeholders involved. Elicitation of requirements can still be regarded as a strong challenge in engineering design because of the high level of uncertainty and the unpredictable factors involved; referred to as unknown unknowns (Sutcliffe and Sawyer, 2013). Significant work has been done in exploring the role of prototypes in requirements elicitation by Jensen et al. (2017). In more detail they examine the use of low and high fidelity prototypes both during and after the concept development design stage as well as their practice in internal and external stakeholder interaction. They note that prototypes are proven to be very beneficial in terms of eliciting users' requirements as far as unknown unknowns are concerned. Also they highlight that prototypes are likewise able to assist in enhancing designers' tacit knowledge during the fuzzy front end of the design process.

\subsection{Prototype purposes}

As it is also mentioned earlier, although the main difference between 'role' and 'purpose' lies in their definitions regarding 'expected function' and 'intended use', these two terms are, of course, strongly connected to each other. Therefore, using the review of the roles found in the literature as a basis, we outline the prototypes' main purposes that arise from the analysis of each of the examined roles. The discussed prototype include many different kinds of 'sub-roles', according to the more specific areas of each role where prototypes are proven to be supportive to designers' needs. For example, as far as their role in learning is concerned, it is noted that prototypes can answer questions regarding the product's functionality and the users' preferences. On the other hand they can be used to communicate a concept to the rest of the team or used for setting milestones and planning the product development process. All these diverse 'sub-roles' can be also referred to as 'functions' of prototypes, according to our definition in Chapter 3. Consequently, the next step is to create a list of Prototype Purposes that derive from these 'functions' by initially developing them further and then merging them accordingly or moving them to a more appropriate role category. Some examples that illustrate the method by which the list is created are listed below:

- Although 'Observation of Interactions' was mentioned as a sub-role of Communication, it was moved to the Requirement Elicitation category considering that it helps in the definition of new and unknown requirements.

- In the case of 'Internal Communication', as long as each of the sub-roles can have an effect either internally (within the design team) or externally (amongst company directors and clients), it was not considered as a purpose.

- In Exploration, divergence and convergence are not included as a separate function as concept generation and concept selection are strongly connected to the processes of expanding generated concepts and consequently narrowing them down.

- Furthermore, some of these functions are not included in our list because they cannot be considered as purposes. In more detail, they are treated as results of the intended use instead; for example, prototypes aid in designers' learning by enhancing their tacit knowledge or through prototyping failure, however designers do not initiate a prototyping activity with these kinds of purposes, therefore they are treated like consequences.

This work results in twenty-three explicit Prototype Purposes. These purposes can also be considered as replies to the question "Why should we build a prototype?" or they can act as guidelines for designers during their prototyping activities. The suggested framework could also potentially function as the basis for the development of a prototyping strategy which presents the prototyping process in a more structured way. This is due to the high importance of setting prototype purposes before starting the prototyping process, as a prototyping strategy is defined as "the planned combination of various techniques to achieve a given set of objectives for a prototyping effort" (Camburn et al., 2017). The list of Prototype Purposes arising from Prototype Roles can be seen in Figure 3 below: 
\# Prototyping Purposes

1 Learning

Answer questions regard ing the product's functionality and technical elements.

Answer questions regarding users' requirements, preferences and behaviours.

Reveal information about unknown factors that may affect performance.

Provide insights to business-related concerns (e.g. manufacturing cost and tooling)

\section{Communication}

Explain a concept to all different stakeholders by demonstrating how it functions.

Communicate aesthetics for stakeholders to experience closely the product's look \& feel.

Get feedback in relation to functionality, performance or aesthetics.

Use the prototype as a means of visual aid for initiating discussion.

Use the prototype as a persuasion tool during design meetings with senior managers.

\section{Refinement}

Identify the product's features with the most significant impact to performance.

Validate specifications and define margins of improvement.

Gather experimental data through a series of testing and optimise performance.

Reveal possible errors during fabrication of the prototype and explore ways of reducing them.

Integration

Evaluate overall aesthetics by developing a whole assembly prototype.

Ensure compatibility between parts and between parts and system.

Configure functionality / performance of the assembly, once all sub-systems are incorporated.

\section{Demonstration}

Set deadlines (milestones) to assist the planning of PD process in terms of time and cost.

Establish forward progress by confirming a certain degree of functionality.

\section{Exploration}

Enhance ideation and develop more concepts through the actual prototyping activity.

Compare the product's characteristics and performance to existing competitor products.

Compare prototypes to each other and proceed to a more informed concept selection.

\section{Elicitation}

Observe users' interactions with the product in order to uncover unknown requirements.

Prioritise requirements and identify the exact target group of users.

Figure 1. List of 23 Prototyping Purposes arising from Prototyping Roles

\section{CONCLUSIONS AND FUTURE WORK}

This paper presents an agenda towards the creation of a new prototype taxonomy through the development of a list of explicit Prototype Purposes. This work is justified from the results of an analysis of eight prototype taxonomies found in the literature. The findings highlight that there is not a taxonomy that explicitly uses the prototypes' purposes as the basic taxonomic dimension. Consequently, the concept of 'Prototype Purpose' is developed by discussing definitions and stating the differences and connections as opposed to the term 'Prototype Role'. After a literature review of both major and less explored prototype roles, the list of prototype purposes is derived from the set of sub-functions of each role.

Regarding future work, since that replies to the question "Why should we build a prototype?" are provided in this paper, the next step is to try and answer "Which kind of prototype should we build for this purpose?" This will be performed by carrying out a mapping between prototypes' characteristics and planned purpose with the aim of developing a new prototype taxonomic classification. This taxonomy can potentially act as a basic tool of a structured prototyping strategy. There is also a need for validating the usability and the accuracy of this proposed taxonomy which will be done by the application of it to design projects in both industrial and academic practices. 


\section{REFERENCES}

Camburn, B., Viswanathan, V., Linsey, J., Anderson, D., Jensen, D., Crawford, R., Otto, K. and Wood, K. (2017), "Design Prototyping Methods: State of the art in Strategies, Techniques, and Guidelines", Design Science, Vol. 3. http://doi.org/10.1017/dsj.2017.10.

Elverum, C.W. and Welo, T. (2014), "The Role of Early Prototypes in Concept Development: Insights from the Automotive Industry", in Procedia CIRP 21, 24th CIRP Design Conference. Elsevier B.V., pp. 491-496. http://doi.org/10.1016/j.procir.2014.03.127.

Gerber, E. and Carroll, M. (2012), "The Psychological Experience of Prototyping”, Design Studies, Elsevier Ltd, Vol. 33 No. 1, pp. 64-84. http://doi.org/10.1016/j.destud.2011.06.005.

Gurjar T.P. (2015), "Effects of a Structured Prototyping Strategy on Capstone Design Projects", Presented to the Faculty of the Graduate School of The University of Texas at Austin, May 2015.

Hess, T. and Summers, J.D. (2013), "Case Study: Evidence of Prototyping Roles in Conceptual Design”, in International Conference on Engineering Design, ICED13, Seoul, Korea, pp. 1-10.

Isa, S.S. and Liem, A. (2014), "Classifying Physical Models and Prototypes in the Design Process: A Study on the Economical and Usability Impact of Adopting Models and Prototypes in the Design Process", in International Design Conference - Design 2014, Dubrovnik - Croatia, pp. 2071-2082.

Jensen, M.B., Elverum, C.W. and Steinert, M. (2017), "Eliciting Unknown Unknowns with Prototypes: Introducing Prototrials and Prototrial-Driven Cultures", Design Studies, Elsevier Ltd, Vol. 49. http://doi.org/10.1016/j.destud.2016.12.002.

Lande, M. and Leifer, L. (2009), 'Prototyping to Learn: Characterizing Engineering Students’ Prototyping Activities and Prototypes", in International Conference on Engineering Design, ICED09, Stanford, CA, USA, pp. 507-516.

Lauff, C.A., Kotys-Schwartz, D. and Rentschler, M.E. (2018), "What is a Prototype? What are the Roles of Prototypes in Companies ?" Journal of Mechanical Design, June 2018. http://doi.org/10.1115/1.4039340.

Lim, Y.-K., Stolterman, E. and Tenenberg, J. (2008), “The Anatomy of Prototypes: Prototypes as Filters , Prototypes as Manifestations of Design Ideas", ACM Transactions on Computer-Human Interaction, 15(2, Article 7). http://doi.org/10.1145/1375761.1375762.

Menold, J., Jablokow, K. and Simpson, T. (2017), "Prototype for X (PFX): A holistic framework for structuring prototyping methods to support engineering design”, Design Studies, Elsevier Ltd, Vol. 50, pp. 70-112. http://doi.org/10.1016/j.destud.2017.03.001.

Michaelraj, A., Hannah, R. and Summers, J.D. (2008), “A Proposed Taxonomy for Physical Prototypes: Structure and Validation”, in ASME 2008 International Design Engineering Technical Conferences \& Computers and Information in Engineering Conference, 2008, New York, USA.

Otto, K. and Wood, K. (2001), Product Design: Techniques in Reverse Engineering and New Product Development, Prentice-Hall.

Pei, E., Campbell, I. and Evans, M. (2015), “A Taxonomic Classification of Visual Design Representations Used by Industrial Designers and Engineering Designers", The Design Journal, p. 6925. http://doi.org/10.2752/175630610X12877385838803.

Stowe, D. (2008), "Investigating the Role of Prototyping in Mechanical Design Using Case Study Validation", https://tigerprints.clemson.edu/all_theses/532.

Sutcliffe, A. and Sawyer, P. (2013), "Requirements Elicitation: Towards the Unknown Unknowns", in Requirements Engineering Conference (RE), 2013 21st IEEE International. http://doi.org/10.1109/RE.2013.6636709.

Ullman (2002), The Mechanical Design Process, McGraw-Hill.

Ulrich, K.T. and Eppinger, S.D. (2012), Product Design and Development, McGraw-Hill. 